\title{
DESIGN OF UNIVERSAL MULTICOSET SAMPLING PATTERNS FOR COMPRESSED SENSING OF MULTIBAND SPARSE SIGNALS
}

\author{
M. E. Domínguez-Jiménez \\ E.T.S.I. Industriales, UPM \\ 28006 Madrid, Spain \\ elena.dominguez@upm.es
}

N. González-Prelcic, G. Vazquez-Vilar, R. López-Valcarce

\begin{abstract}
Many problems in digital communications involve wideband radio signals. As the most recent example, the impressive advances in Cognitive Radio systems make even more necessary the development of sampling schemes for wideband radio signals with spectral holes. This is equivalent to considering a sparse multiband signal in the framework of Compressive Sampling theory. Starting from previous results on multicoset sampling and recent advances in compressive sampling, we analyze the matrix involved in the corresponding reconstruction equation and define a new method for the design of universal multicoset codes, that is, codes guaranteeing perfect reconstruction of the sparse multiband signal.
\end{abstract}

Index Terms - Multicoset sampling, compressive sampling, multiband sparse signal, TI-ADC, universal pattern.

\section{INTRODUCTION}

Recent interest in Cognitive Radio (CR) systems [1] motivate sensing schemes for multiband sparse signals which allow the CR to decide which channels are really occupied and, in consequence, make an efficient usage of the spectrum. At the same time, intensive work in the area of compressive sampling has yielded a number of algorithms and hardware systems to obtain compressed samples from frequency-domain sparse signals. Taking into account that CR systems need to analyze wide frequency bands, any scheme that could acquire the full wide band at a sampling rate lower than the corresponding Nyquist rate could provide samples to an spectrum analyzer based on compressed measurements $[2,3]$ with the consequent reduction in resource usage.

Most hardware systems which have been proposed for the acquisition of compressed samples are based on Time-

Work supported by the European Regional Development Fund (ERDF) and the Spanish Government under projects TEC2009-08133, TEC201021245-C02-02/TCM and CONSOLIDER-INGENIO 2010 CSD2008-00010, and the Galician Regional Government under projects "Consolidation of Research Units" 2009/62 and 2010/85.

\author{
E.E. Telecomunicación, Universidade de Vigo \\ 36313 Vigo, Spain \\ \{nuria,gvazquez,valcarce\}@gts.uvigo.es
}

Interleaved Analog-to-Digital Converters (TI-ADCs) ${ }^{1}$. Different sampling patterns to operate different TI-ADC architectures have been reported, e.g., in [5, 6, 7, 8]. All these works assume that the wideband signal is multiband sparse (i.e., only a small number of frequency subbands are occupied) and that the locations of the occupied subbands are not known a priori.

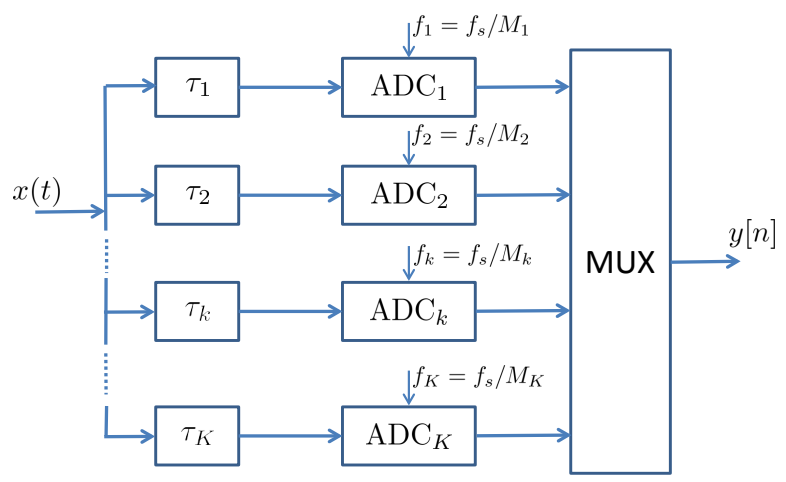

Fig. 1. Block diagram of a general TI-ADC system.

Fig. 1 shows the structure of a general TI-ADC system, where $f_{s}=1 / T$ denotes the Nyquist rate, $M_{i} \in \mathbb{Z}$, and $\tau_{i}$ are different delays obtained as $\tau_{i}=\left(c_{i} / d_{i}\right) T, i=1, \ldots, K$ with $c_{i}, d_{i} \in \mathbb{Z}$. The most common usage of TI-ADCs is a particular case of this model, with $\tau_{i}=(i-1) T$ and equal sampling frequencies $f_{i}=f_{s} / K$. The general structure of Fig. 1 include the different sampling strategies proposed in the aforementioned papers. These are briefly reviewed next.

In [5], a $p$-sparse multiband signal is assumed. The uniform sampling grid (at Nyquist rate) is divided into blocks of $L$ consecutive samples, and then only $p$ out of these $L$ samples are acquired. This can be implemented with only $p$ branches of the TI-ADC system in Fig. 1, using $\tau_{i}=c_{i} T$ with integers $0 \leq c_{0}<c_{1}<\cdots<c_{p} \leq L-1$ and equal sampling frequencies $f_{i}=f_{s} / L$. Only certain selections of the $p$ output

\footnotetext{
${ }^{1}$ A noteworthy exception is the modulated wideband converter proposed in [4].
} 
channels lead to the reconstruction of the original signal, although the authors of [5] do not provide a design criterion for this; the issue of finding an appropriate set $\left\{c_{i}\right\}_{i=1}^{p}$ (a multicoset code) is stated to be a combinatorial problem.

Another TI-ADC sampling scheme was proposed in [7], although the focus was on the reconstruction algorithm and not on the issue of channel selection; this is accomplished as in [9]. The same authors proposed later [6] a set of TI-ADCs working synchronously (i.e., with $\tau_{i}=0, i=0, \ldots, K-$ 1 ), and using different sampling frequencies for each ADC (with the sum of of these different sampling rates lower than the Nyquist rate). Again, the focus is on the development of a reconstruction scheme for a set of different and arbitrarily chosen sampling frequencies; no criterion is proposed on how to choose the sampling frequencies. Note that selecting a set of sampling frequencies is equivalent to selecting a specific multicoset code in the architecture of [5].

The scheme proposed in [8] can also be implemented with the TI-ADC architecture of Fig. 1 and a multicoset sampling scheme, with a random selection of the output channels. The main drawback of random selection approaches is that they require to have the $K \mathrm{ADCs}$ constantly working. In contrast, fixed channel selection methods allow to reduce the number of branches of the hardware system to the number of active bands $p$, with the corresponding reduction in area size and power consumption. Motivated by this fact, we provide an algorithm for the design of universal multicoset codes, i.e., for the selection of the parameters in the general TI-ADC system of Fig. 1 guaranteeing perfect reconstruction of $p$-sparse multiband signals. To our knowledge, this is the first systematic method proposed for this goal; previous codes were either obtained by direct search [10] or relied on the choice of a prime value of $L$, which renders every pattern universal [5].

\section{PROBLEM STATEMENT}

As in [5], we assume a complex-valued $p$-sparse multiband signal $x(t)$, bandlimited to $\left[0, f_{s}\right]$. The number of active bands is $N$, and an upper bound $B$ for the bandwidth of each of these bands is known. The sampling stage is implemented as in Fig. 1 by considering $K=L, \tau_{k}=k T$ and $f_{k}=f_{s} / L$ for $k=1, \ldots, L$. Only $p \geq N$ ADC channels are implemented. Thus, for each block of $L$ Nyquist-rate samples, $p$ of them are acquired, with indices $0 \leq c_{1}<c_{2}<\ldots c_{p} \leq L-1$. This can also be seen as obtaining $p$ different uniform sampling sequences $x_{c_{i}}[n]$ at rates $f_{s} / L$ :

$$
x_{c_{i}}[n]= \begin{cases}x(t=n T), & n=m L+c_{i} \text { for some } m \in \mathbb{Z} \\ 0, & \text { otherwise }\end{cases}
$$

Following [5], we define the sampling pattern $C=$ $\left\{c_{i}\right\}_{i=1}^{p}$. The reconstruction of the multiband signal from the acquired samples requires the selection of $L, p$ and $C$ such that $X(f)$ can be reconstructed based on

$$
\boldsymbol{y}(f)=\frac{1}{L T} \boldsymbol{A} \boldsymbol{x}(f)
$$

with

$$
\begin{aligned}
y_{i}(f) & =X_{c_{i}}\left(e^{j 2 \pi f T}\right), \quad i=1, \ldots, p, \\
x_{k}(f) & =X\left(f+\frac{k}{L T}\right), \quad k=0, \ldots, L-1,
\end{aligned}
$$

and where $\boldsymbol{A}$ is a $p \times L$ matrix with elements

$$
a_{i k}=\exp \left(j \frac{2 \pi}{L} c_{i} k\right)
$$

It is noted in [5] that $\boldsymbol{x}(f)$ can be recovered from $\boldsymbol{y}(f)$ if $L \leq f_{s} / B, p \geq N$ and $\boldsymbol{A}$ has full Kruskal-rank ${ }^{2}$, with this rank being equal to $p$. For given $L$ and $p$, a sampling pattern $C$ that results in a fully Kruskal-rank $\boldsymbol{A}$ is termed universal [5]. In the remainder several results are presented revealing certain properties of the Kruskal-rank of $\boldsymbol{A}$, which in turn will suggest a design method for universal sampling patterns.

\section{K-RANK ANALYSIS OF VANDERMONDE MATRICES}

Let us define $w=e^{j \frac{2 \pi}{L}}$ and

$$
w_{i}=\exp \left(j \frac{2 \pi}{L} c_{i}\right)=w^{c_{i}}, \quad i=1, \ldots, p .
$$

Then, from (5), one has $a_{i k}=w_{i}^{k}$. Thus, the $i$-th row of $\boldsymbol{A}$ contains consecutive powers of $w_{i}$ : $\left[1, w_{i}, w_{i}^{2}, \cdots, w_{i}^{L-1}\right]$. In other words, the rows of $\boldsymbol{A}$ are extracted from those of an $L \times L$ row-wise Vandermonde matrix. Although there is no general criterion for the K-rank of a row-wise Vandermonde matrices, the following result applies to column-wise Vandermonde matrices.

Lemma 1. Consider $p$ distinct numbers $x_{1}, \ldots, x_{p} \in \mathbb{C}$. Let $\boldsymbol{V}$ be the $n \times p$ column-wise Vandermonde matrix defined as

$$
v_{i j}=x_{j}^{i-1}, \quad i=1, \ldots, n, \quad j=1, \ldots, p .
$$

Then the K-rank of $\boldsymbol{V}$ is equal to its rank, which is $\min (p, n)$.

Proof. For $n=p$, recall that the Vandermonde square matrix of order $p$ has full rank iff the $p$ numbers $x_{1}, \ldots, x_{p}$ are distinct, which is the case; hence the K-rank of $\boldsymbol{V}$ equals its rank $p$. If $p<n$, the $p$ columns of $\boldsymbol{V}$ are linearly independent since the we can build a $p \times p$ submatrix from the first $p$ rows of $\boldsymbol{V}$; as this square submatrix is also a column-wise Vandermonde matrix of $p$ distinct numbers $x_{1}, \ldots, x_{p}$, then it has full rank $p$, so the K-rank of $\boldsymbol{V}$ is $p$.

\footnotetext{
${ }^{2}$ The Kruskal- or K-rank of a matrix is the largest value of $m$ such that every subset of $m$ columns of the matrix is linearly independent.
} 
Finally for $p>n$, let us consider any set of $n$ columns of $\boldsymbol{V}$. These form another column-wise Vandermonde matrix, whose entries are the powers of $x_{m_{1}}, \ldots, x_{m_{n}}$. As these $n$ numbers are distinct, its rank is maximum $(n)$. Hence the $\mathrm{K}$ rank of $\boldsymbol{V}$ is $n$.

Notice that the maximum value for the K-rank (or the rank) of a $p \times n$ matrix is $\min (p, n)$. Hence, Lemma 1 gives a sufficient condition for $\boldsymbol{V}$ to have maximum K-rank. Let us prove that it is also necessary.

Corollary 2. Let $\boldsymbol{V}$ be a $n \times p$ column-wise Vandermonde matrix whose columns are the powers of $x_{1}, \ldots, x_{p} \in \mathbb{C}$. Then

$$
K \text {-rank of } \boldsymbol{V} \text { is maximum } \Longleftrightarrow x_{1}, \ldots, x_{p} \text { are distinct. }
$$

Proof. On one hand, if $x_{i}=x_{k}$ then the $i$-th and $k$-th columns of $\boldsymbol{V}$ coincide, so its K-rank is 1 (not maximum since $p, n>1)$. On the other hand, if all the numbers $x_{1}, \ldots, x_{p}$ are distinct, Lemma 1 ensures that the K-rank of $\boldsymbol{V}$ is maximum.

\section{DESIGN OF UNIVERSAL MULTICOSET CODES}

From the previous results it is possible to provide conditions on $c_{1}, c_{2}, \ldots, c_{p}$ under which $\boldsymbol{A}$ has maximum K-rank $(p)$.

Theorem 3. Let $c_{1}, c_{2}, \ldots, c_{p}(p \leq L)$ constitute an arithmetic progression of difference $d \neq 0$ (i.e., $c_{i}=c_{1}+$ $(i-1) d)$. Let $\boldsymbol{A}$ be the $p \times L$ row-wise Vandermonde matrix defined by (5). Then

\section{$\boldsymbol{A}$ has maximum $K$-rank $(p) \Longleftrightarrow d$ and $L$ are coprime.}

Proof. Matrix $\boldsymbol{A}$ can be written as

$$
\boldsymbol{A}=\left(\begin{array}{rrrrr}
1 & w_{1} & w_{1}^{2} & \cdots & w_{1}^{L-1} \\
1 & w_{2} & w_{2}^{2} & \cdots & w_{2}^{L-1} \\
\vdots & \vdots & & \cdots & \vdots \\
1 & w_{p} & w_{p}^{2} & \cdots & w_{p}^{L-1}
\end{array}\right)
$$

Now we use that $c_{i}=c_{1}+(i-1) d$. Each $w_{i}=w^{c_{i}}=$ $w^{c_{1}+(i-1) d}=w_{1} w^{(i-1) d}$. Hence $\boldsymbol{A}$ takes the form

$$
\left(\begin{array}{rrrrr}
1 & w_{1} & w_{1}^{2} & \cdots & \\
1 & w_{1} w^{d} & w_{1}^{2} w^{2 d} & \cdots & w_{1}^{L-1} w^{(L-1) d} \\
\vdots & \vdots & & \cdots & \\
1 & w_{1} w^{(p-1) d} & w_{1}^{2} w^{2(p-1) d} & \cdots & w_{1}^{L-1} w^{(L-1)(p-1) d}
\end{array}\right)
$$

By extracting the factor $w_{1}^{j-1}$ from the $j$-th column, $\boldsymbol{A}$ is seen to have the same K-rank as the matrix

$$
\left(\begin{array}{rrrrr}
1 & 1 & 1 & \cdots & 1 \\
1 & w^{d} & w^{2 d} & \cdots & w^{(L-1) d} \\
\vdots & \vdots & & \cdots & \vdots \\
1 & w^{(p-1) d} & w^{2(p-1) d} & \cdots & w^{(L-1)(p-1) d}
\end{array}\right)
$$

which is indeed a $p \times L$ column-wiseVandermonde matrix of numbers $1, w^{d}, w^{2 d}, \cdots, w^{(L-1) d}$. As $p \leq L$, Corollary 2 states that its K-rank is maximum $(p)$ iff all those $L$ numbers are distinct. Let us show that two of these numbers are equal iff $d$ and $L$ are not coprime. Indeed, there exist $0 \leq a<$ $b<L$ such that $w^{a d}=w^{b d}$, or equivalently $w^{(b-a) d}=1$, iff $(b-a) d=c L$ for some integer $c$. This amounts to saying that $d$ and $L$ are not coprime. The claim follows.

The following sufficient condition now follows.

Corollary 4. Any set of consecutive $p$ numbers $c_{1}, \ldots, c_{p}$ (with $p \leq L$ ), yield a matrix $\boldsymbol{A}$ of maximum $K-\operatorname{rank}(p)$.

Proof. Consecutive numbers form an arithmetic progression of difference $d=1$, which is always coprime with $L$. So the claim follows from Theorem 3 .

Remark 5. Note that the difference $d$ can be either positive or negative, and smaller or greater than $L$. The proof does not make use of the assumption $0<d<L$. Hence, the numbers $c_{1}, c_{2}, \ldots, c_{p}$ need not be in increasing order (as stated in [5]). Moreover, they can even be greater than $L$; in that case they would be replaced by $c_{j} \bmod L$. That would yield different sets of numbers $c_{1}, c_{2}, \ldots, c_{p}$ with no order at all (neither increasing nor decreasing).

\subsection{Examples}

- For $L=12$, difference $d=5$ yields, for $c_{1}=0$, the arithmetic progression $0,5,10,3,8,1,6,11,4,9,2,7,0, \ldots$ (cyclic repetition of length 12) where we have substituted $c_{j}$ by $c_{j} \bmod 12$ where necessary. Theorem 3 ensures that every subset of $p \leq 12$ consecutive numbers of this sequence would yield maximum K-rank. For instance, $(5,10,3)$ or its permutation $(3,5,10)$ or its shifts, such as $(0,2,7)$, which is equivalent to its permutation $(2,7,0)$ which is the end of that length-12 cycle.

- For $L=12$, difference $d=7$ yields, for $c_{1}=0$, the arithmetic progression $0,7,2,9,4,11,6,1,8,3,10,5,0, \ldots$. which is the reversed version of the sequence in the previous example. For $c_{1}=2$ we would obtain $2,9,4,11,6,1,8,3,10,5,0,7$ which is a shifted arithmetic sequence.

- For $L=12$, the sequence $(1,3,8)$ yields maximum Krank since it appears as $3,8,1$ in the previous sequence of difference $d=5$.

To conclude this section let us recall the meaning of the designed universal patterns in terms of the parameters of the TI-ADC system in Fig. 1: for a TI-ADC architecture working with equal sampling frequencies $f_{k}=f_{s} / L$, selecting $c_{i}$ as proposed in Theorem 3 means selecting the $p$ output branches with $\tau_{i}=c_{i} T, i=1, \ldots, p$. 


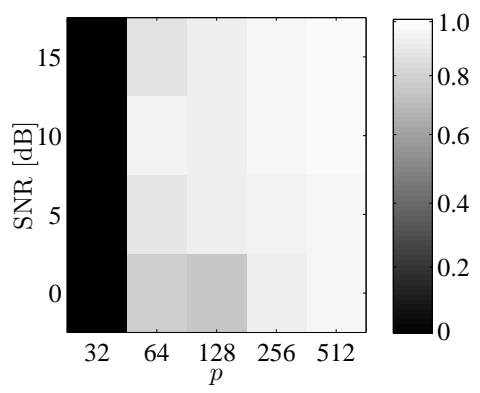

(a)

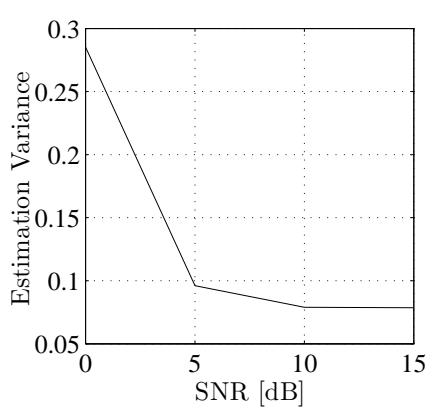

(b)
Fig. 2. PSD estimation performance. (a) Support recovery probability. (b) Estimator Variance for $p=128$.

\section{EXPERIMENTAL RESULTS IN A CR FRAMEWORK}

We illustrate the effectiveness of the proposed multicoset sampling scheme in the context of the spectral estimation algorithm for multichannel wideband signals presented in $[3]^{3}$. This algorithm reconstructs the power spectral density (psd) of the received signal from a set of compressed measurements, which are acquired by the TI-ADC scheme previously discussed.

The psd to be estimated consists of a set of channels which are $8 \mathrm{MHz}$ wide and can be occupied by signals with flat spectrum with bandwidth $B=7.61 \mathrm{MHz}$. We assume that only 3 of the channels are occupied, and for simplicity in the presentation of results, they are all received with the same power when active.

Fig. 2 shows the performance of the proposed estimator for a range of signal-to-noise ratio (SNR) and different values of the compression factor. Here we assume $L=1024$, while $p$ ranges from 32 to 512 , i.e. a compression factor from $1 / 32$ to $1 / 2$. In Fig. 2(a) we can observe a good recovery performance for compression factors larger that $1 / 16$, even when the SNR presents a finite value. On the other hand, Fig. 2(b) shows the variance of the power level estimator versus the SNR for fixed $p=128$. Comparing this figure with Fig. 2(a) we can see that the variance is greatly reduced when the right support is determined.

\section{CONCLUSIONS}

We have analyzed the Kruskal-rank of the reconstruction matrix in a multicoset sampling framework, assuming an input multiband signal and without a priori knowledge of band locations. From this analysis we have derived our main result: a method to design universal multicoset sampling patterns, that

\footnotetext{
${ }^{3}$ Compression matrix of dimension $p \times L$, 4 sample covariance matrix realizations are averaged as input to the algorithm, 100 Monte Carlo realizations for each simulated point.
}

is, which guarantee perfect reconstruction of the multiband signal within the framework of compressive sampling. In addition, we have presented some experimental results within a Cogntive Radio setting, showing the effectiveness of the designed sampling patterns.

\section{REFERENCES}

[1] B. Wang and K. J. R. Liu, "Advances in cognitive radio networks: A survey," IEEE J. Sel. Topics Signal Process., vol. 5, pp. 5-23, Feb. 2011.

[2] Y. Lamelas-Polo, Y. Wang, A. Pandharipande, and G. Leus, "Compressive wideband spectrun sensing," in IEEE Int. Conf. Acoust., Speech, Signal Process. (ICASSP), 2009.

[3] G. Vazquez-Vilar, R. López-Valcarce, C. Mosquera, and N. González-Prelcic, "Wideband spectral estimation from compressed measurements exploiting spectral a priori information in cognitive radio systems," in IEEE Int. Conf. Acoust., Speech, Signal Process. (ICASSP), 2010.

[4] M. Mishali and Y. C. Eldar, "From theory to practice: Sub-nyquist sampling of sparse wideband analog signals," IEEE J. Sel. Topics Signal Process., vol. 4, pp. 375-391, Apr. 2010.

[5] M. Mishali and Y. C. Eldar, "Blind multiband signal reconstruction: Compressed sensing for analog signals," IEEE Trans. Signal Process., vol. 57, pp. 993-1009, Mar. 2009.

[6] M. Fleyer, A. Linden, M. Horowitz, and A. Rosenthal, "Multirate synchronous sampling of sparse multiband signals," IEEE Trans. Signal Process., vol. 58, pp. 1144-1156, Mar. 2010.

[7] A. Rosenthal, A. Linden, and M. Horowitz, "Multirate asynchronous sampling of sparse multiband signals," $J$. Optical Soc. Amer., vol. 25, pp. 2320-2330, Sep. 2008.

[8] J. Laska et al., "Random sampling for analog to information conversion of wideband signals," in IEEE Dallas Circuits Syst. Workshop (DCAS), 2006.

[9] R. Venkataramani and Y. Bresler, "Optimal sub-Nyquist nonuniform sampling and reconstruction for multiband signals," IEEE Trans. Signal Process., vol. 49, pp. 2301-2313, Oct. 2001.

[10] R. Venkataramani and Y. Bresler, "Perfect reconstruction formulas and bounds on aliasing error in subNyquist nonuniform sampling of multiband signals," IEEE Trans. Inf. Theory, vol. 46, pp. 2173-2183, Sep. 2000. 